\author{
MILITARY TEEHNIGAAL COLLEGE \\ CAIRC - EGYPT
}

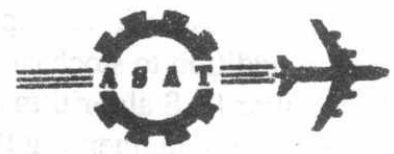

$7^{\text {th }}$ INTERNATIONAL CONF. ON AEROSPACE SCIENCES \& AVIATION TECHNOLOGY

\title{
ARMAX Augmented UD Identification Algorithm (AUDIX)
}

\author{
Gamal A. El-Sheikh ${ }^{\star}$
}

\begin{abstract}
:
An ARMAX augmented UD identification (AUDIX) algorithm for systems identification is developed to identify an ARMAX model by rearranging the regressor and parameter vectors and augmenting the covariance matrix of Bierman's UD factorization algorithm. The structure of the AUDIX is particularly easy to interpret and it is a direct extension of the augmented UD identification (AUDI) which is a direct extension to the Recursive Least Squares (RLS) algorithm. The proposed algorithm permits simultaneous identification of model parameters, disturbed by a noise model, plus loss functions for all orders from $l$ to $n$ at each step with approximately the same calculation effort as $\mathrm{n}^{\text {th }}$ order RLS, in addition it has a fast convergence rate than the AUDI. Simply, this AUDIX algorithm is a least-squares estimator, with the same numerical properties as those of Bierman's UD factorization algorithm. In addition, its structure and implementation are more straightforward and easier to analyze than the UD algorithm. Therefore, the AUDIX provides a convenient and efficient basis to be used on-line in Seif-Tuning or adaptive control algorithms. This is because in real applications no guarantee that it will be free from disturbances and measurement noises in addition to stochastic environments. Thus, the objective is to estimate the parameters of the model which best fit a set of observed or measured data. This model is time-varying and is used to determine the parameters of the controller to cope with the changing process characteristics. This algorithm is utilized with the self tuning control of an aeroengine and proved robustness to numerical singularities in addition to fast and good tracking. The paper contains some of the results with a general example showing the good convergence and tracking for this algorithm.
\end{abstract}

Keywords: Systems Identification, Recursive Techniques, UD Factorization, Adaptive Control.

\section{1- Introduction:}

Most adaptive systems are based, explicitly or implicitly, on recursive identification $[5,6,8,16]$. That is Recursive Parameter Estimation (RPE) techniques are required when a process model is to be constructed based on a limited set of data which lead to the availability of an estimated model of the process at all times. Therefore, the problem is that: given a set of observed data/variables and it is required to estimate the parameters of the model which best fit these data in some sense given that the model structure is predefined. This time-varying model is used to determine the parameters of the \{also time-varying\} regulator/controller. In this way the regulator will be dependent on the previous behavior of the process and it should adapt to the changing process characteristics.

The parameter estimates of a dynamic system may be computed using either On-Line methods which compute the parameter estimates recursively in time i.e. if there is an estimate $\theta(t-1)$ based on data up to time $(t-1)$, then $\theta(t)$ is computed by simple modification of $\theta(t-1)$, or Off-Line or Batch methods in which all the recorded data are used simultaneously to find the parameters' estimates. The field of system identification is filled with limitless number of different algorithms, the choice between them depends on some factors among them are model complexity, noise-to-signal ratio, convergence rate, and computational expense. Recursive Least Squares (RLS) is the most widely used parameter estimation algorithm because of its conceptual simplicity, ease of implementation and relatively fast convergence rate [1,2,4,9]. However, the basic RLS has also a number of shortcomings such as poor numerical performance. One of the most successful algorithms for improved numerical performance is the Bierman's UD factorization algorithm $[1,9,14,15]$. However, this algorithm is not widely used due to its complexity for interpreting and implementing. Therefore, it is extended by rearranging the order of the regressor and parameter vectors and augmenting the covariance matrix to yield the ARMAX augmented UD identification algorithm (AUDIX). 
The idea of this paper is motivated due to the need for ARMAX models in Self-Tuning process and the poor results obtained using the AUDI algorithm. This is because in real applications no guarantee that it will be free from disturbances and measurement noises in addition to stochastic environments. The paper shows that the UD algorithm can be directly related to the familiar RLS algorithm and can be easily extended by rearranging the order of the regressor and parameter vectors and augmenting the covariance matrix. This rearrangement yields the proposed ARMAX augmented UD identification algorithm (AUDLX) which

1. Simultaneously estimates the parameters and loss functions for all ARMAX model orders from 1 to $n$; where $\mathrm{n}$ is a user specified upper limit for the model order; with approximately the same computational effort as $n^{\text {th }}$ order RLS,

2. is inherently a least-squares algorithm,

3. has good numerical properties and stability,

4. is much easier to interpret and understand than the original UD algorithrn.

\section{2- Problem Formulation:}

A dynamical system, with the configuration shown in Fig. 1, can be described by the following linear difference equation:

$$
A\left(z^{-1}\right) y(t)=B\left(z^{-1}\right) u(t)+C_{d}\left(z^{-1}\right) \zeta(t)
$$

where; $u(t)$ represents input signal to the system, $y(t)$ represent output signal from the system, and $\zeta(t)$ represents some disturbance acting on the system output. The system polynomials are defined as follows:

$$
\begin{array}{ll}
A\left(z^{-1}\right) & =1+a_{1} z^{-1}+\ldots \ldots .+a_{n_{b}} z^{-n_{a}} \\
B_{k}\left(z^{-1}\right) & =b_{1}+b_{2} z^{-1}+\ldots \ldots \ldots+b_{n_{b}} z^{-n_{b}} \\
B\left(z^{-1}\right) & =z^{-k} B_{k} \\
C_{d}\left(z^{-1}\right) & =1+c_{1} z^{-1}+\ldots \ldots .+c_{n_{c}} z^{-n_{c}} \\
n_{a} & =\operatorname{deg}(A) \\
n_{b} & =\operatorname{deg}\left(B_{k}\right) \quad ; b_{1} \neq 0 \quad \& \quad k \geq 1 \\
n_{c} & =\operatorname{deg}\left(C_{d}\right)
\end{array}
$$

This system is known as the ARMAX model in which $\zeta(t)$ is modeled as a moving average (MA) of a serially uncorrelated white noise sequence. It can be put in the following vector form

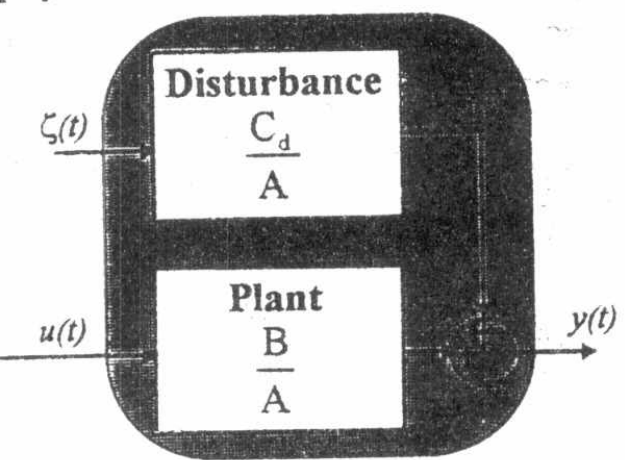

Fig. 1: System model in ARMAX form $y(t)=\theta^{T} \phi(t)+\zeta(t)$ where the variable $\theta$ denotes the parameter vector formed according to the following form:

$$
\theta=\left[a_{1} a_{2} a_{3} \cdots \cdots \cdots a_{n_{2}} b_{1} b_{2} \cdots \cdots \cdots b_{n_{b}} c_{1} c_{2} \cdots \cdots \cdots c_{n_{c}}\right]^{T}
$$

The variable $\phi$ represents the regression/signal vector defined as

$$
\begin{aligned}
\phi=[-y(t-1)-y(t-2) & \cdots \cdots \cdots-y\left(t-n_{a}\right) \\
u(t-k) u(t-k-1) \cdots \cdots \cdots u\left(t-k-n_{b}\right) & \\
& \left.\zeta(t-1) \zeta(t-2) \cdots \cdots \cdots\left(t-n_{c}\right)\right]^{T}
\end{aligned}
$$

This model is not linear regression since $\phi(t)$ contains the unobserved data $\zeta(t)$. Applying any of the available identification methods to the model in $\mathrm{Eq}^{\mathrm{n}}$ (3) yields estimates of the parameter vector $\theta$. That is, estimates for the system polynomials $\mathrm{A}\left(\mathrm{z}^{-1}\right), \mathrm{B}\left(\mathrm{z}^{-1}\right)$ and $\mathrm{C}_{\mathrm{d}}\left(\mathrm{z}^{-1}\right)$.

\section{3- The Recursive Least Squares Method (RLS)}

Recalling the model (3), $y(t)=\theta^{\mathrm{T}} \phi(t)+\zeta(t)$, with the definitions of $\theta$ and $\phi$ as in (4) and (5) and the disturbance term $\zeta(t)$ is not specified, the objective is to obtain estimates of the parameter vector $\theta$ from measurements of $y(t)$ and $\phi(t)$. Since $\zeta(\mathrm{t})$ in $\mathrm{Eq}^{\mathrm{n}}(3)$ is not specified, a prediction of $y(t)$ given the previous data values can be obtained according to the relation:

$\hat{y}(t / \theta)=\phi^{T}(t) \hat{\theta}(t-1)$ 
Let us define $\varepsilon(t)$ as the prediction error, which is the difference between the measured output $y(t)$ and the one-step ahead prediction of $y(t)$ made at time $(t-1)$ and based on the model corresponding to the estimate $\hat{\theta}(t-1)$. Thus, if $\varepsilon(t)$ is small, the estimate $\hat{\theta}(t-1)$ is good and should not be modified very much. This prediction error is thus given by the relation:

$\varepsilon(\mathrm{t}) \equiv \mathrm{y}(\mathrm{t})-\hat{\mathrm{y}}(\mathrm{t} / \theta)=\mathrm{y}(\mathrm{t})-\phi^{\mathrm{T}}(\mathrm{t}) \hat{\theta}(\mathrm{t}-1)$

A natural way to obtain estimates of $\theta$ is to try to minimize the prediction error (7), w.r.t. $\theta$, using the following criterion $[11,13]$ :

$\mathrm{V}_{\mathrm{N}}(\theta)=\frac{1}{\mathrm{~N}} \sum_{1}^{\mathrm{N}}\left\{\mathrm{y}(\mathrm{t})-\phi^{\mathrm{T}}(\mathrm{t}) \hat{\theta}(\mathrm{t}-1)\right\}^{2}$

This criterion is quadratic in $\theta$ and can therefore be minimized analytically to yield the estimate $[4,11]$

$\hat{\theta}(N)=\left[\sum_{t=1}^{N} \phi(t) \phi^{T}(t)\right]^{-1} \cdot\left[\sum_{t=1}^{N} \phi(t) y(t)\right]$

or

$\hat{\theta}(t)=\left[\sum_{j=1}^{t} \lambda_{f}^{t-j} \phi(j) \phi^{T}(j)\right]^{-1} \cdot\left[\sum_{j=1}^{t} \lambda_{f}^{t-j} \phi(j) y(j)\right]$

This is the non-recursive least-squares estimate of the parameter $\theta$. For real-time applications the recursive form of the estimate, called RLS algorithm $[3,6,7,8,9,15]$, will be as follows:

$$
\begin{aligned}
& \hat{\theta}(\mathrm{t})=\hat{\theta}(\mathrm{t}-1)+\mathrm{K}(\mathrm{t}) \varepsilon(\mathrm{t}) \\
& \varepsilon(\mathrm{t})=\mathrm{y}(\mathrm{t})-\phi^{\mathrm{T}}(\mathrm{t}) \hat{\theta}(\mathrm{t}-1) \\
& \mathrm{K}(\mathrm{t})=\frac{\mathrm{P}(\mathrm{t}-1) \phi(\mathrm{t})}{\lambda_{\mathrm{f}}+\phi^{\mathrm{T}}(\mathrm{t}) \mathrm{P}(\mathrm{t}-1) \phi(\mathrm{t})} \\
& \mathrm{P}(\mathrm{t})=\mathrm{P}(\mathrm{t}-1)-\frac{\mathrm{P}(\mathrm{t}-1) \phi(\mathrm{t}) \phi^{\mathrm{T}}(\mathrm{t}) \mathrm{P}(\mathrm{t}-1)}{\lambda_{\mathrm{f}}+\phi^{\mathrm{T}}(\mathrm{t}) \mathrm{P}(\mathrm{t}-1) \phi(\mathrm{t})} \\
& \varepsilon_{\mathrm{r}}(\mathrm{t})=\mathrm{y}(\mathrm{t})-\phi^{\mathrm{T}}(\mathrm{t}) \hat{\theta}(\mathrm{t})
\end{aligned}
$$

where; $\lambda_{\mathrm{f}}$ is the forgetting factor, $\varepsilon_{\mathrm{r}}(\mathrm{t})$ is the residual error of the parameter estimates and $\mathrm{K}(\mathrm{t})$ is a weighting or kalman gain factor showing how much the value of $\varepsilon(t)$ will modify the different elements of the parameter vector. The form of the kalman-gain factor given in $\mathrm{eq}^{\mathrm{n}}(11)$ is more appropriate or convenient to be implemented in computation and updating of the covariance matrix $P(t)$. The covariance matrix $P(t)$ is given by the relation [11]:

$P(t)=\left[\sum_{t=1}^{N} \phi(t) \phi^{T}(t)\right]^{-1}$

This matrix is called so because its expectation is proportional to the covariance matrix of the parameter estimates. The corresponding loss function has the following recurive form

$$
\begin{aligned}
J(t) & =\sum_{j=1}^{t} \lambda_{f}^{t-j} \varepsilon_{r}^{2}(j) \\
& =\lambda_{f} J(t-1)+\varepsilon_{r}(t) \varepsilon(t)
\end{aligned}
$$

This algorithm gives unbiased estimates [11] \{i.e. estimates tend to the true parameters at steady storo \} only if $\zeta(\mathrm{t})$ and $\phi(\mathrm{t})$ are uncorrelated, which holds when $\zeta(\mathrm{t})$ is a white noise sequence. However, the covarinnce matrix $P(t)$ is updated from $P(t-1)$ using the potentially unstable formula $\mathrm{Eq}^{\mathrm{n}}(11)$ which makes the violation or the positive-definiteness-property of $P(t)$ probable. So for achieving more numerical robustness, Bierman [1] proposed the UD factorization algorithm, described shortly next in this paper.

\section{4- Bierman's UD factorization algorithm}

Bierman's UD factorization algorithm uses a different method for updating the matrix $P(t)$ that greatly improves the numerical properties of $P(t)$. This is done by decomposing the covariance matrix $P(t)$ into $P(t)=U(t) D(t) U^{T}(t)$ form, where $U(t)$ and $D(t)$ are a unit-upper-triangular matrix and a diagonal 
matrix, respectively. Then, the matrices $\mathrm{U}(\mathrm{t})$ and $\mathrm{D}(\mathrm{t})$, instead of $P(t)$, are updated at every time step. This decomposition guarantees the positive-definiteness of the covariance matrix $P(t)$, and thus higher identification accuracy and numerical robustness can be obtained $[1,6,9,12]$. This algorithm in form of steps can be described as follows:

Step-1: A.t time $t=0$, set $\mathrm{P}(0)=\mathrm{U}(0) \mathrm{D}(0) \mathrm{U}^{\mathrm{T}}(0)=\sigma^{2} \mathrm{I}$, with $\sigma$ is of large integer-value, or accurately set $D(0)=\sigma^{2} I$, and $U(0)=I$. Then, update $U(t-1)$ and $D(t-1)$ to yield $\mathrm{U}(\mathrm{t})$ and $\mathrm{D}(\mathrm{t})$, respectively, using Steps $2-8$

Step-2: $\quad$ Construct the regressor vector $\phi(t)$ as in Eqn (5), then compute

$\mathrm{f}=\mathrm{U}^{\mathrm{T}}(\mathrm{t}-1) \phi(\mathrm{t})$

$g=D^{T}(t-1) f$

$\beta_{\mathrm{o}}=\lambda_{\mathrm{f}}(\mathrm{t})$

Step-3: $\quad$ For $\mathrm{j}=1, \cdots \cdots, \mathrm{d}$; go through Steps 4-6; where $d=3 n$ is the dimension of the regressor vector,

$\mathrm{U}(\mathrm{t})$ and $\mathrm{D}(\mathrm{t})$.

Compute the following variables/matrices

$$
\begin{array}{ll}
\beta_{j} & =\beta_{j-1}+f_{j} g_{j} \\
D_{j j}(t) & =\frac{\beta_{j-1} D_{j i}(t-1)}{\beta_{j} \lambda_{f}(t)} \\
V_{j} & =g_{j} \\
\mu_{j} & =-\frac{f_{j}}{\beta_{j-1}}
\end{array}
$$

Step -5 : $\quad$ For $i=1, \cdots \cdots, j-1$; do Step-6 $\{$ if $j=1$, skip Step-6 $\}$

Step -6 : Compute the following variables/matrices

$$
\begin{aligned}
& U_{i j}(t)=U_{i j}(t-1)+V_{i} \mu_{j} \\
& V_{i}=V_{i}+U_{i j}(t-1) V_{j}
\end{aligned}
$$

Step-7: Compute the kalman gain factor

$$
\begin{aligned}
& \hat{\mathrm{K}}(\mathrm{t})=\left[\mathrm{V}_{1} \mathrm{~V}_{2} \cdots \cdots \mathrm{V}_{\mathrm{d}}\right]^{\mathrm{T}} \\
& \mathrm{K}(\mathrm{t})=\frac{\hat{\mathrm{K}}(\mathrm{t})}{\beta_{\mathrm{d}}}
\end{aligned}
$$

Step-8: $\quad$ Update the parameter vector $\hat{\theta}(\mathrm{t})$ as

$$
\hat{\theta}(\mathrm{t})=\hat{\theta}(\mathrm{t}-1)+\mathrm{K}(\mathrm{t}) \varepsilon(\mathrm{t})
$$

\section{5- AUDIX algorithm}

The AUDI algorithm, used to estimate the parameters of the ARX model, is described in Niu et al. [12]. The proposed AUDIX algorithm can be started by rearranging the regressor and parameter vectors in $\mathrm{Eq}^{\mathrm{ns}}(4,5)$ as follows:

1. The regressor vector $\psi_{\mathrm{a}}(\mathrm{t})$ is formulated as follows$$
\psi_{\mathrm{a}}(\mathrm{t})=\left[-\mathrm{y}(\mathrm{t}-\mathrm{n}), \mathrm{u}(\mathrm{t}-\mathrm{k}-\mathrm{n}), \varepsilon_{\mathrm{r}}(\mathrm{t}-\mathrm{n}), \cdots \cdots \cdots,-\mathrm{y}(\mathrm{t}-1), \mathrm{u}(\mathrm{t}-\mathrm{k}), \varepsilon_{\mathrm{r}}(\mathrm{t}-1)\right]^{\mathrm{T}}
$$

and the augmented regressor vector $\phi_{\mathrm{a}}(\mathrm{t})$ will be as follows

$\phi_{\mathrm{a}}(\mathrm{t})=\left[-\mathrm{y}(\mathrm{t}-\mathrm{n}), \mathrm{u}(\mathrm{t}-\mathrm{k}-\mathrm{n}), \varepsilon_{\mathrm{r}}(\mathrm{t}-\mathrm{n}), \cdots \cdots \cdot,-\mathrm{y}(\mathrm{t}-\mathrm{l}), \mathrm{u}(\mathrm{t}-\mathrm{k}), \varepsilon_{\mathrm{r}}(\mathrm{t}-1),-\mathrm{y}(\mathrm{t})\right]^{\mathrm{T}}$

$$
=\left[\psi_{3}^{\mathrm{T}}(\mathrm{t})-\mathrm{y}(\mathrm{t})\right]^{\mathrm{T}}
$$

The regressor vector is rearranged and the output $y(t)$ is added in order to simplify the formulation and interpretation of the AUDIX algorithm, where the error/noise sequence $\zeta(t)$ is predicted by the residual error $\varepsilon_{\mathrm{r}}(\mathrm{t})$. 
2. The new parameter vector $\hat{\theta}_{a}(t)$ will be rearranged in a similar manner as follows:

$\hat{\theta}_{a}(t)=\left[a_{n} b_{n} c_{n} a_{n-1} b_{n-1} c_{n-1} \cdots \cdots \cdots a_{1} b_{1} c_{1}\right]^{T}$

where the subscript $n$ represents the assumed model order, $\hat{\phi}_{\mathrm{a}}(\mathrm{t})$ represents the augmented regressor vector whose dimension is $(3 n+1)$ or $d$. Now the new covariance matrix $\mathrm{P}_{\mathrm{a}}(\mathrm{t})$ will have the form:

$P_{a}(t)=\left[\sum_{j=1}^{t} \lambda_{f}^{t-j} \phi_{a}(j) \phi_{a}^{T}(j)\right]^{-1}$

which is decomposed into the form $P_{a}(t)=U_{a}(t) D_{a}(t) U_{a}^{T}(t)$; where

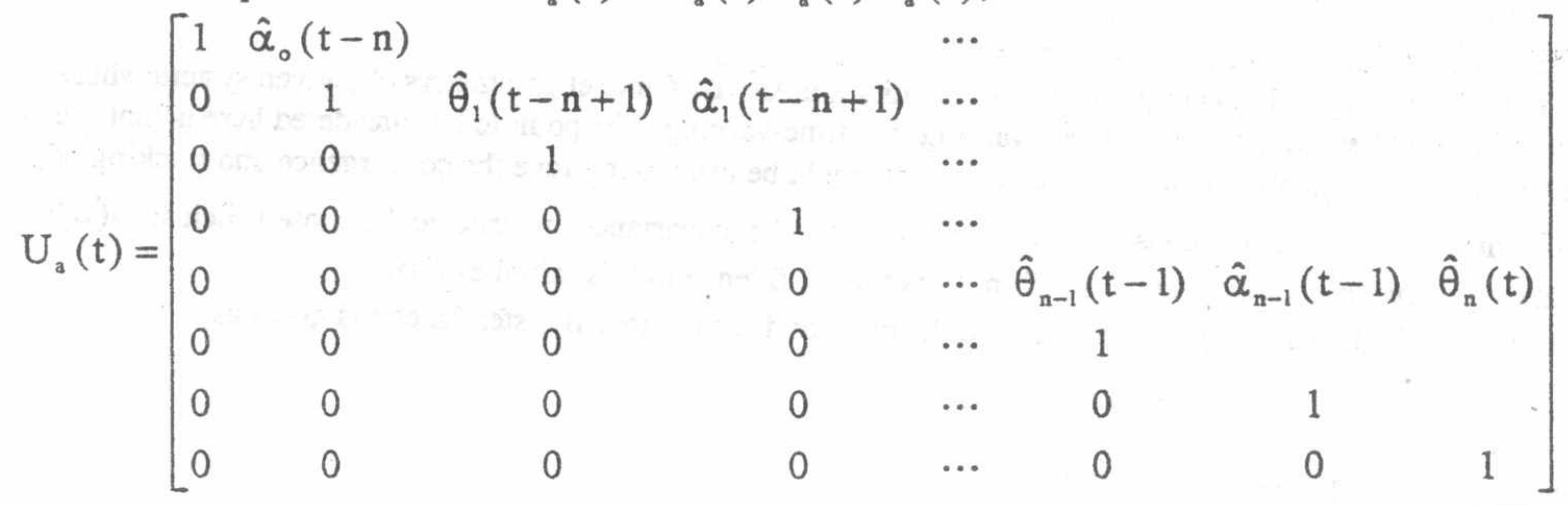

and

$D_{a}^{-1}(t)=\operatorname{diag}\left[J_{0}(t-n), L_{0}(t-n), \cdots \cdots \cdots \cdot J_{n-1}(t-1), L_{n-1}(t-1), J_{n}(t)\right]$

In Bierman's UD algorithm, the matrices $U$ and $D$ are used to update the kalman gain factor $K(t)$ and then the parameter vector $\hat{\theta}_{a}(t)$ is updated recursively as in the conventional RLS. However, in the presented AUDIX algorithm, $\mathrm{U}_{\mathrm{a}}(\mathrm{t})$ and $\mathrm{D}_{\mathrm{a}}(\mathrm{t})$ matrices already contain the parameter estimates and the loss functions for all model orders from $l$ to $n$. Therefore, the AUDIX algorithm can be implemented through the following steps:

Step-1: $\quad$ At time $t=0$, set $\mathrm{P}_{\mathrm{a}}(0)=\mathrm{U}_{\mathrm{a}}(0) \mathrm{D}_{\mathrm{a}}(0) \mathrm{U}_{\mathrm{a}}^{\mathrm{T}}(0)=\sigma^{2} \mathrm{I}$, with $\sigma$ is of large integer-value, or accurately set $D_{a}(0)=\sigma^{2} I$, and $U_{a}(0)=I$. Then, update $U_{a}(t-1)$ and $D_{a}(t-1)$ to yield $U_{a}(t)$ and $D_{a}(t)$, respectively, using Steps $2-6$

Step-2: $\quad$ Construct the regressor vector $\phi_{\mathrm{a}}(\mathrm{t})$ as in $\mathrm{Eq}^{\mathrm{n}}$ (18), then compute

$$
\begin{aligned}
& \mathrm{f}=\mathrm{U}_{\mathrm{a}}^{\mathrm{T}}(\mathrm{t}-1) \phi_{\mathrm{a}}(\mathrm{t}) \\
& \mathrm{g}=\mathrm{D}_{\mathrm{a}}^{\mathrm{T}}(\mathrm{t}-1) \\
& \beta_{\mathrm{o}}=\lambda_{\mathrm{f}}(\mathrm{t})
\end{aligned}
$$

Step-3: $\quad$ For $\mathrm{j}=1, \cdots \cdots, \mathrm{d}$; go through Steps 4-6; where $d=3 n+l$ is the dimension of the regressor vector, $\mathrm{U}_{\mathrm{a}}(\mathrm{t})$ and $\mathrm{D}_{\mathrm{a}}(\mathrm{t})$.

Step-4: $\quad$ Compute the following variables/matrices

$$
\begin{aligned}
\beta_{j} & =\beta_{j-1}+f_{j} g_{j} \\
D_{a_{j j}}(t) & =\frac{\beta_{j-1} D_{a_{j j}}(t-1)}{\beta_{j} \lambda_{f}(t)} \\
V_{j} & =g_{j} \\
\mu_{j} & =-\frac{f_{j}}{\beta_{j-1}}
\end{aligned}
$$

Step-5: $\quad$ For $i=1, \cdots \cdots, j-1$; do Step-6 $\{$ if $j=1$, skip Step-6 $\}$

Step-6: Compute the following variables/matrices 


$$
\begin{array}{ll}
U_{a_{i j}}(t) & =U_{a_{i j}}(t-1)+V_{i} \mu_{j} \\
V_{i} & =V_{i}+U_{a_{i j}}(t-1) V_{j}
\end{array}
$$

Note that in this algorithm Steps 7-8 of Bierman's UD algorithm had been omitted and paid for by the inclusion of the current output $y(t)$ in the regressor vector $\hat{\phi}_{\mathrm{a}}(\mathrm{t})$. The updated parameter vector $\hat{\theta}_{\mathrm{a}}(\mathrm{t})$ is included in the last column of the $U_{a}$ matrix.

\section{6- Numerical implementation/evaluation}

Now the AUDIX algorithm is implemented to identify the ARMAX model parameters of a given system whose dynamics are either constant or slowly varying or time-varying. The point to be considered here is that the forgetting factor $\left(\lambda_{f}\right)$, either constant or exponential, could be used to improve the convergence and tracking of the algorithm. The initial values for the estimate and the covariance matrix can be chosen such as $\hat{\theta}(0)$ indicates the a priori estimate of $\theta$ and $P(0)$ reflects the confidence in this initial estimate.

Example-1: Consider the system model with the plant and disturbance transfer functions given as

$$
\begin{aligned}
& W_{p} \equiv \frac{B}{A}=\frac{z^{-1}\left(1+0.5 z^{-1}\right)}{1-1.5 z^{-1}+0.7 z^{-2}} \\
& W_{d} \equiv \frac{C_{d}}{A}=\frac{\left(1-1.4 z^{-1}-0.48 z^{-2}\right)}{1-1.5 z^{-1}+0.7 z^{-2}}
\end{aligned}
$$

Where, the input signal $u(t)$ is a Pseudo-Random-Binary-Sequence (PRBS) with amplitude \pm 1 , the disturbance $\zeta(t)$ is a white noise with zero mean and 0.02 variance, using the variables $\lambda_{\mathrm{f}}(0)=0.99, \lambda_{\mathrm{f}}=0.97$, $\mathrm{P}_{\mathrm{a}}(0)=10^{4} \mathrm{I}$ and model order $n=3$ or 2 . The evolution of parameter estimates using the AUDI is shown in Fig. 2, where the algorithm did not consider any disturbances, and using the AUDIX is shown in Fig. 3, where disturbance model is considered. The difference between the two algorithms is clear where the AUDIX converges very fast and gives good tracking in addition it is the appropriate algorithm to be utilized with adaptive control and can simulate the real process applications.

\section{Conclusions:}

The proposed AUDIX algorithm is a least-squares estimator, with the same numerical properties as those of Bierman's UD factorization algorithm. It provides simultaneous estimates of the parameters and loss functions for all model orders from 1 to $n$ with the same computational effort required for the $n^{\text {th }}$ order RLS. The structure and implementation of the AUDIX as well as AUDI algorithm are more straightforward and easier to analyse than the UD algorithm. The AUDIX provides a convenient and efficient basis to be used on-line in Self-Tuning control and/or algorithms in addition to the fast convergence than the AUDI. The AUDIX presented in this paper could be used with a general ARMAX models where the parameter estimates of the $1^{\text {st }}, 2^{\text {nd }}, 3^{\text {td }}, 4^{\text {th }}, \cdots \cdots$ order models are in $4^{\text {th }}, 7^{\text {th }}, 10^{\text {th }}, \cdots \cdots$ columns of the U-matrix, instead of $3^{\text {rd }}, 5^{\text {th }}, 7^{\text {th }}, \cdots \cdots$ columns of the U-matrix in the AUDI. The updated parameter vector $\hat{\theta}_{\mathrm{a}}(\mathrm{t})$ is included in the $(3 n+1)^{\text {th }}$ column of the $U_{a}$ matrix.

\section{References}

[1] Bierman, G.J., Factorization Methods for Discrete Sequential Estimation, Academic Press, New York, 1977.

12] Bittanti, S., P.Bolzern and M. Campi, Convergence and Exponential Convergence of Identification Algorithms with Directional Forgetting Factor, Automatica, Vol. 26, No. 5, pp 929-932, 1990.

[3] Chen and J.P. Norton, Estimation Technique for Tracking Rapid Parameter Changes, Int. J. Control, Vol. 45, No. 4, 1987, pp 1387-1398.

[4] Dstrom, K.J., and P. Eykhoff, System Identification: A Survey, Automatica, Vol. 7, pp 123-162, 1971.

[5]El-Sheikh, G.A., M.J. Grimble and M.A. Johnson, On the Performance of GH $H_{\infty}$ Self-Tuning for AeroEngine Control, Control'94, Warwick University, March 21-24, 1994a.

[6] El-Sheikh, M.A. Johnson, and M.J. Grimble, Explicit Self-Tuning Control Using Generalized Cost Functions, Third International Conference on Computer Applications in Industry, Cairo, Egypt, Dec. 26-29, $1994 \mathrm{~b}$.

7] Favier, G. and D. Dubois, A Review of K-Step-Ahead Predictors, Automatica, Vol. 26, No. 1, pp 75-84, 1990.

[8] Goodwin, G.C. and K.S. Sin, Adaptive Filtering, Prediction and Control, Prentice Hall, 1984. 
[9] Greene, M.E. and T.S. Denney, Real-Time Estimator for Control of an Orbiting Single Tether System, IEEE Transactions on Aexospace and Electronic Systems, Vol. 27, No. 6, November 1991.

[10]Gustavsson, L. Ljung and T. Soderstrom. Survey Paper: Identification of Processes in Closed Loop Identifiability and Accuracy Aspects, Automatica, Vol. 13, pp 59-75, 1977.

[11]Ljung, L. and T. Soderstrom, Theory and Practice of Recursive Identification, The MIT Press, 1983.

[12]Niu, S., D.G. Fisher and Deyun Xiao, An Augmented UD Identification Algorithm, Int. J. Control, Vol. 56, No. 1, pp 193-211, 1992.

[13] Soderstrom, T. and P. Stoica, System Identification, Prentice Hall, 1989. [14]Weinfeld, R, Tow

[15]Wellstead, P.E. and P. Zanker, Techniques of Self-Tuning, Optimal Control Applications and Methods, Vol.3, No.4, pp305-322, Oct-Dec 1982.

[16]Wellstead, P.E. and M.B. Zarrop, Self-Tuning Systems: Control and Signal Processing, John Wiley \& Sons, UK, 1991.

[17]Zhang, H.G., J.Chen, and H.Y.Zhang, A Recursive Identification Algorithm for Estimating Abrubt Parameter Changes, IEE International Conference Control 1991, number 332, Vol.1
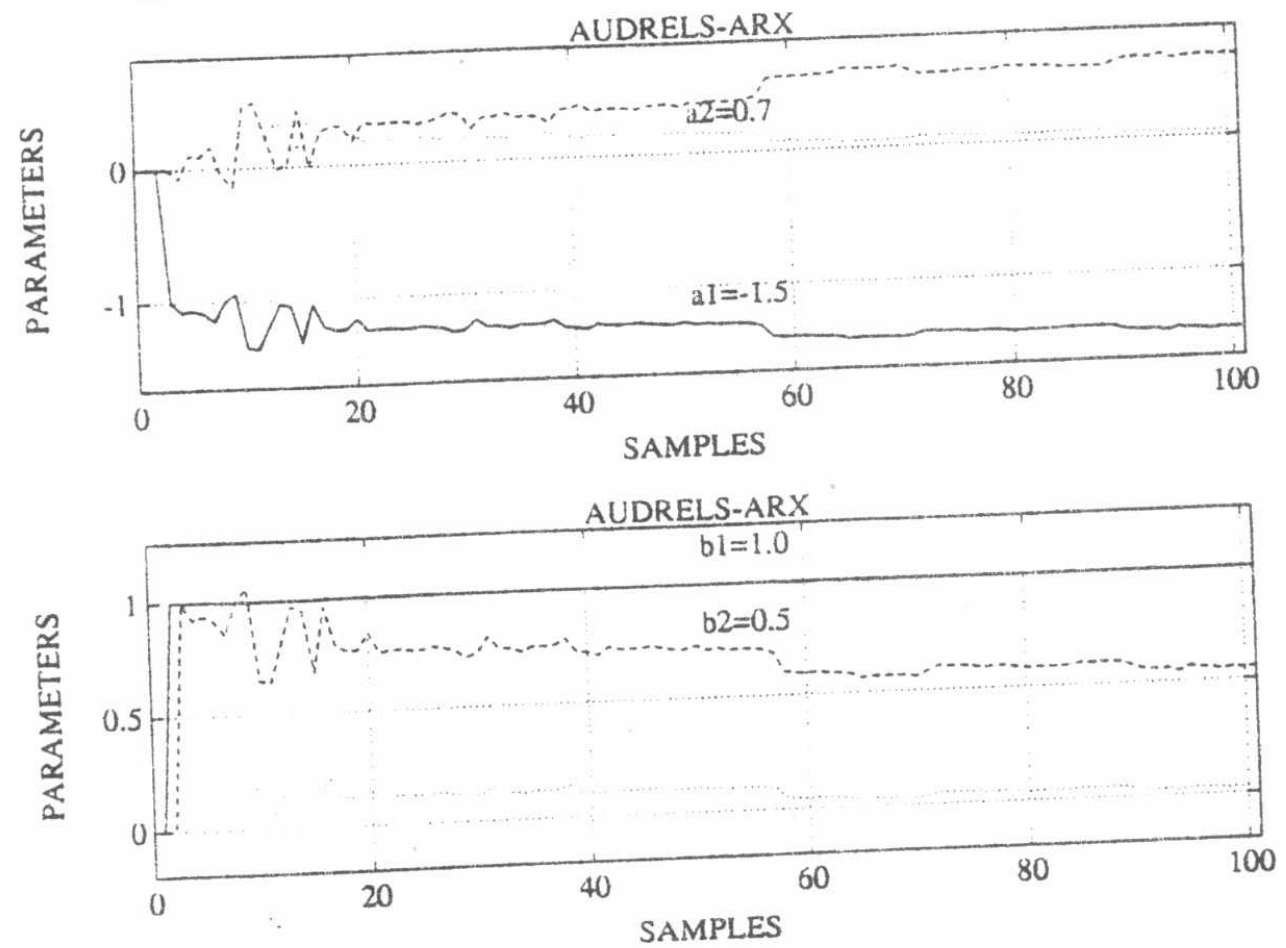

Fig. 2 :Parameter estimates using the AUDI
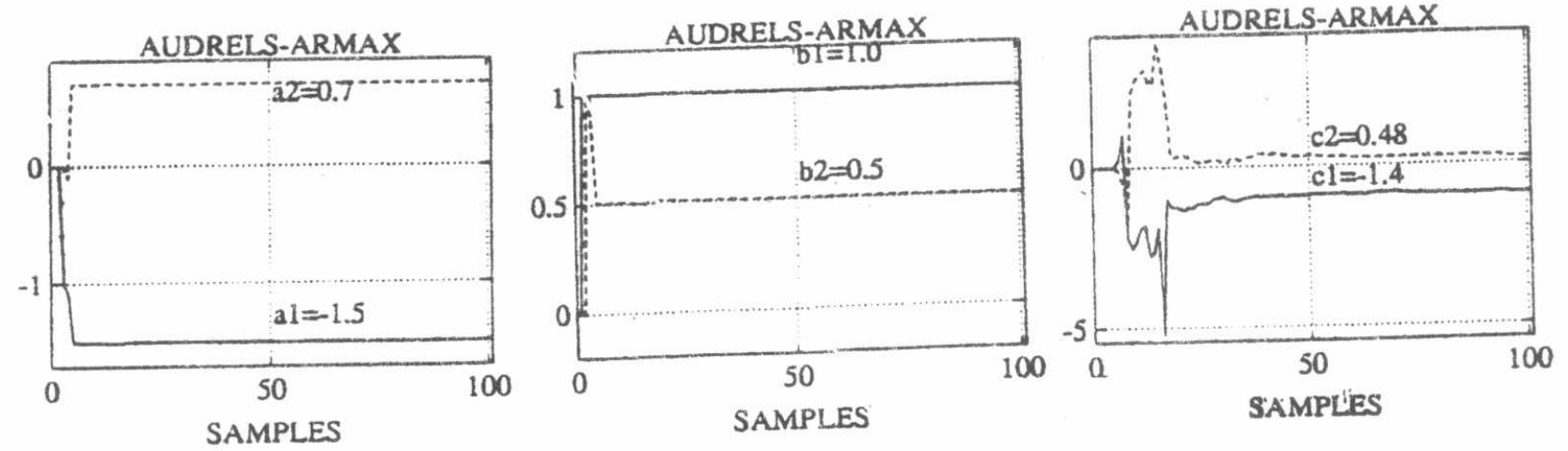

Fig. 3 :Parameter estimates using the AUDDX 